OPEN ACCESS

Edited by:

Mitsuo Tagaya,

Tokyo University of Pharmacy and Life

Sciences, Japan

Reviewed by:

Xiangping Chu,

University of Missouri-Kansas City,

United States

Stephan Kellenberger,

University of Lausanne, Switzerland

*Correspondence:

Feihu Chen

chenfeihu@ahmu.edu.cn

Specialty section:

This article was submitted to

Membrane Traffic,

a section of the journa

Frontiers in Cell and Developmental

Biology

Received: 19 August 2020

Accepted: 05 October 2020

Published: 23 October 2020

Citation:

Xu Y and Chen F (2020) Factors

and Molecular Mechanisms

Influencing the Protein Synthesis,

Degradation and Membrane

Trafficking of ASIC1a.

Front. Cell Dev. Biol. 8:596304.

doi: 10.3389/fcell.2020.596304

\section{Factors and Molecular Mechanisms Influencing the Protein Synthesis, Degradation and Membrane Trafficking of ASIC1a}

\author{
Yayun $\mathrm{Xu}^{1,2,3}$ and Feihu Chen ${ }^{1,2,3 *}$ \\ ${ }^{1}$ Department of Epidemiology and Biostatistics, School of Public Health, Anhui Medical University, Hefei, China, ${ }^{2}$ The Key \\ Laboratory of Major Autoimmune Diseases of Anhui Province, Anhui Institute of Innovative Drugs, School of Pharmacy, Anhui \\ Medical University, Hefei, China, ${ }^{3}$ The Key Laboratory of Anti-inflammatory and Immune Medicines, Ministry of Education, \\ Hefei, China
}

Acid-sensing ion channels (ASICs) are members of the degenerin/epithelial sodium channel superfamily. They are extracellular $\mathrm{pH}$ sensors that are activated by protons. Among all ASICs, ASIC1a is one of the most intensively studied isoforms because of its unique ability to be permeable to $\mathrm{Ca}^{2+}$. In addition, it is considered to contribute to various pathophysiological conditions. As a membrane proton receptor, the number of ASIC1a present on the cell surface determines its physiological and pathological functions, and this number partially depends on protein synthesis, degradation, and membrane trafficking processes. Recently, several studies have shown that various factors affect these processes. Therefore, this review elucidated the major factors and underlying molecular mechanisms affecting ASIC1a protein expression and membrane trafficking.

Keywords: factors, membrane trafficking, molecular mechanisms, protein expression, acid-sensing ion channel 1a

\section{INTRODUCTION}

As a member of the degenerin/epithelial sodium channel (DEG/ENaC) superfamily, acid-sensing ion channel 1a (ASIC1a) senses $\mathrm{pH}$ changes and has an extensive distribution pattern and function in the peripheral tissues and central nervous system (Radu et al., 2016). ASIC1a is sensitive to extracellular acidification and is involved in several acidosis-related pathophysiological processes, including inflammation (Duan et al., 2007), hypoxia (Tan et al., 2011), and pain (Deval et al., 2010). Plasma membrane expression is critical to the function of ASICs that act as extracellular proton sensors (Waldmann and Lazdunski, 1998). Protein synthesis and degradation, as well as dynamic trafficking processes partially determine the number and function of receptors present on the plasma membrane (Zeng et al., 2014). Therefore, elucidating the factors and the underlying molecular mechanisms that affect ASIC1a expression and membrane trafficking will improve our understanding of its pathophysiological functions in multiple diseases. 


\section{FACTORS AND MOLECULAR MECHANISMS INFLUENCING PROTEIN SYNTHESIS, DEGRADATION, AND MEMBRANE TRAFFICKING OF ASIC1a}

Several studies have demonstrated that protein synthesis, degradation, and membrane trafficking of ASIC1a are affected by various factors. Since the number of ASIC1a present on the cell surface correlates with its total expression (Wu et al., 2016), the presence of the channel in the cytoplasm can be used as a pool for supplying to the membrane. Therefore, it is of great significance to explore the factors that influence both the total expression and membrane trafficking of ASIC1a. These factors are highlighted in Table 1.

The number of receptors present on the plasma membrane partly depends on protein synthesis, degradation, and dynamic trafficking processes. Among them, dynamic trafficking processes mainly include sorting and forward trafficking of the surface receptors from the endoplasmic reticulum through the Golgi apparatus to the plasma membrane via endocytosis and exocytosis (Zeng et al., 2014). An in-depth understanding of the mechanisms that affect the cell surface expression of ASIC1a is essential for a better understanding of cell signal transduction under acidic conditions (Yang et al., 2012). Therefore, we have provided the details of the underlying molecular mechanisms that govern ASIC1a protein synthesis, degradation, and dynamic trafficking (Figure 1).

\section{Acidosis and Hypoxia}

ASIC1a has been characterized as a potent proton sensor for detecting extracellular acidification in the peripheral tissues and brain (Cheng et al., 2018). Our previous studies have indicated that extracellular acidosis increases protein expression of ASIC1a in a $\mathrm{pH}$ - and time-dependent manner in rat articular chondrocytes (Dai et al., 2017; Gao et al., 2019; Zu et al., 2020). Moreover, it has been reported that ASIC1a expression is significantly increased in the articular cartilage of adjuvant arthritis (AA) rats (Zhou et al., 2015), the articular synovial fluid of which showed a low $\mathrm{pH}$ value compared to that of the non-arthritic rat synovial fluid (Zhou et al., 2018). Furthermore, blocking ASIC1a expression through pretreatment with the ASIC1a-specific blocker such as psalmotoxinf (PcTx1) or ASIC1a RNA interference (RNAi) reversed the promoting effect of extracellular acidification on the protein and mRNA expression levels of ASIC1a and decreased cell death induced by extracellular acidosis (Gao et al., 2019). Similarly, after PcTx1 treatment, both the severity of disease in AA rats and the protein expression of ASIC1a in the synovial tissue decreased in vivo (Qian et al., 2020). These results suggest that the injury caused by extracellular acidification might be related to the upregulation of ASICla expression.

Hypoxia triggers pathological processes by producing an acidic microenvironment in multiple diseases (Damgaci et al., 2018). Additionally, an increasing number of studies suggest that pathological hypoxia can alter the activity of ASICs. This might provide a progressive understanding of the hypoxic effects in
TABLE 1 | The factors that regulate the total expression and membrane trafficking of ASIC1a.

\begin{tabular}{|c|c|c|c|}
\hline Factors & $\begin{array}{c}\text { Total } \\
\text { expression }\end{array}$ & $\begin{array}{l}\text { Membrane } \\
\text { trafficking }\end{array}$ & References \\
\hline Acidosis & $\uparrow$ & & $\begin{array}{l}\text { Dai et al., 2017; Gao et al., } \\
\text { 2019; Zu et al., } 2020\end{array}$ \\
\hline Hypoxia & $\uparrow$ & & $\begin{array}{c}\text { Tan et al., 2011; Jernigan } \\
\text { et al., } 2012\end{array}$ \\
\hline \multicolumn{4}{|c|}{ Inflammatory cytokines } \\
\hline IL-6 & $\uparrow$ & & Zhou et al., 2015 \\
\hline $\mathrm{IL}-18$ & $\uparrow$ & & Zhou et al., 2018 \\
\hline $\mathrm{TNF}-\alpha$ & $\uparrow$ & & Zhou et al., 2018 \\
\hline \multicolumn{4}{|l|}{ Neurotrophins } \\
\hline NGF & $\uparrow$ & & $\begin{array}{c}\text { Matricon et al., 2013; Wei } \\
\text { et al., } 2020\end{array}$ \\
\hline PDGF & $\uparrow$ & $\uparrow$ & Zuo et al., 2019 \\
\hline BDNF & & $\uparrow$ & Duan et al., 2012 \\
\hline \multicolumn{4}{|l|}{ Hormones } \\
\hline$\beta$-estradiol & $\downarrow$ & & $\begin{array}{c}\text { Zhou et al., 2019a; Song } \\
\text { et al., } 2020\end{array}$ \\
\hline Insulin & $\downarrow$ & & Chai et al., 2010 \\
\hline \multicolumn{4}{|l|}{ Drugs } \\
\hline Ibuprofen & $\downarrow$ & & Sun et al., 2014 \\
\hline Aspirin & $\downarrow$ & & Wu et al., 2019 \\
\hline Omeprazole & $\uparrow$ & & Thongon et al., 2014 \\
\hline Amiloride & $\downarrow$ & & Sun et al., 2014 \\
\hline PcTx1 & $\downarrow$ & $\downarrow$ & $\begin{array}{l}\text { Duan et al., 2012; Gao } \\
\text { et al., 2019; Wu et al., } 2019\end{array}$ \\
\hline Ginsenoside-Rd & $\downarrow$ & & Zhang et al., 2012 \\
\hline \multicolumn{4}{|l|}{ MicroRNAs } \\
\hline miR-149 & $\downarrow$ & & Jiang and Zha, 2017 \\
\hline miR-144 & $\downarrow$ & & Jiang and Zha, 2017 \\
\hline let-7 & $\uparrow$ & & Jiang and Zha, 2017 \\
\hline \multicolumn{4}{|l|}{ Effector proteins } \\
\hline $\mathrm{p} 11$ & & $\uparrow$ & Donier et al., 2005 \\
\hline PICK1 & & $\uparrow$ & Jin et al., 2010 \\
\hline Rho & & $\uparrow$ & Herbert et al., 2018 \\
\hline SGK-1 & & $\downarrow$ & Arteaga et al., 2008 \\
\hline AP2 & & $\downarrow$ & Zeng et al., 2013 \\
\hline Dynamin1 & & $\downarrow$ & Zeng et al., 2013 \\
\hline \multicolumn{4}{|l|}{ Chemicals } \\
\hline Acute ethanol & $\downarrow$ & & Zhou et al., 2019b \\
\hline $\mathrm{H}_{2} \mathrm{O}_{2}$ & & $\downarrow$ & Zha et al., 2009 \\
\hline NO & $\begin{array}{c}\mathrm{PFC} \uparrow, \\
\text { hippocampus } \downarrow\end{array}$ & & Li et al., 2019 \\
\hline N-glycosylation & & $\uparrow$ & Jing et al., 2012 \\
\hline
\end{tabular}

cancer, arthritis, and ischemic brain injury (Yingjun and Xun, 2013). The expression and function of ASICla were upregulated after hypoxia in cultured retinal ganglion cells, and PcTx1 was able to reduce cell death in vitro (Tan et al., 2011). These results indicate that ASIC1a activation plays a role in cell death induced by hypoxia. Additionally, exposure to chronic hypoxia upregulated ASIC1 protein expression in pulmonary arterial smooth muscle. Furthermore, PcTx1 prevented enhanced store-operated $\mathrm{Ca}^{2+}$ entry in pulmonary vascular smooth muscle (Jernigan et al., 2012), suggesting that upregulation of 


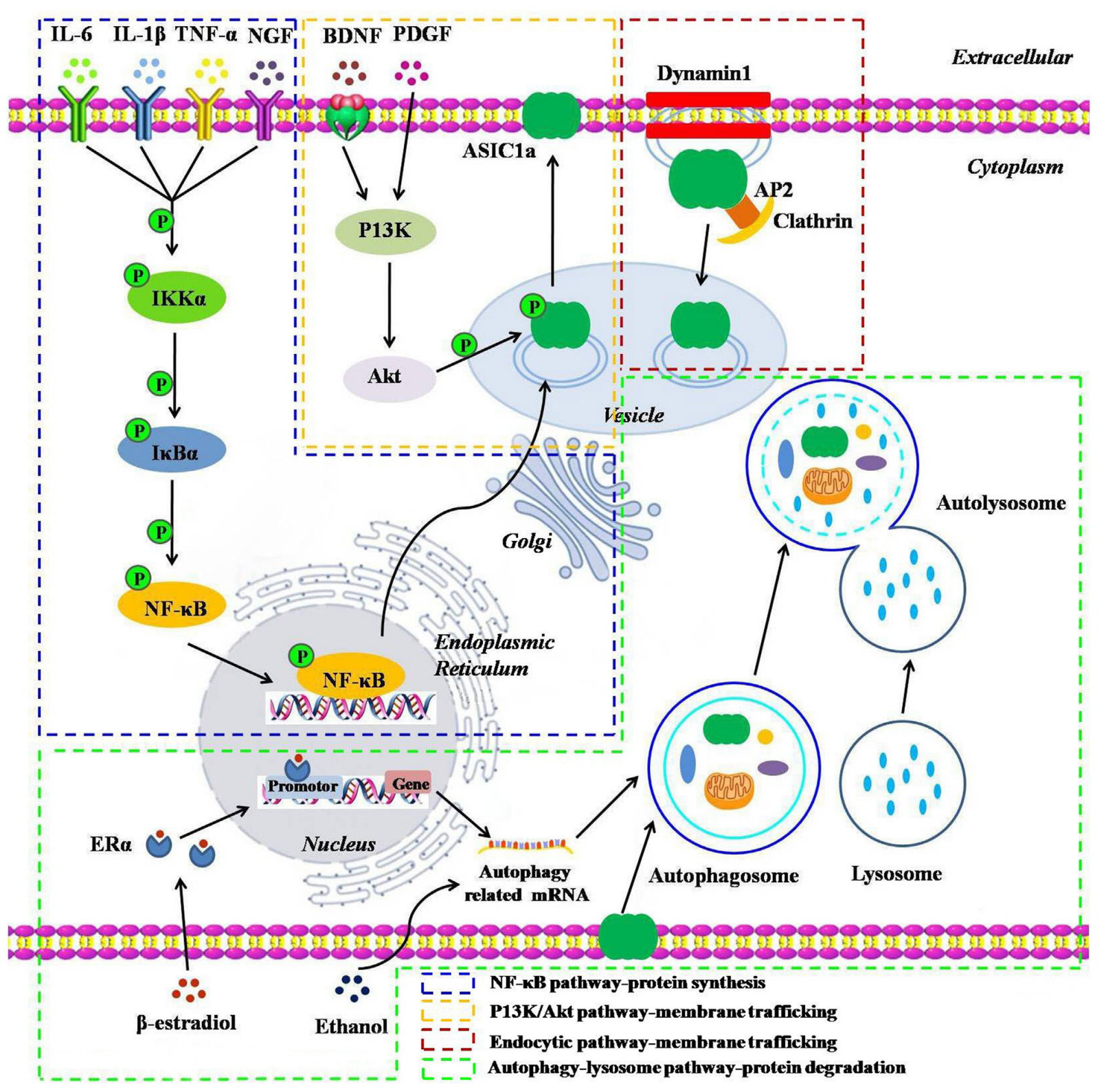

FIGURE 1 | A schematic representation of the underlying molecular mechanisms influencing the protein synthesis, degradation, and dynamic trafficking processes of ASIC1a. The NF-kB pathway: inflammatory cytokines including IL-6, IL-1 $\beta$, TNF- $\alpha$, and NGF and their receptor interactions activate the NF-kB pathways, leading to the translocation of NF-KB p65 into the nucleus and enhancing the ASIC1a gene promoter activity, thereby upregulating ASIC1a expression; The PI3K/Akt pathway: BDNF/TrkB or PDGF activates the intracellular PI3K/Akt pathway, and then, induces ASIC1a phosphorylation in vesicle and forward targeting to the plasma membrane; The endocytic pathway: downregulation of ASIC1a surface expression in a clathrin- and dynamin-dependent endocytosis; Autophagy-lysosome pathway: $\beta$-estradiol/ER $\alpha$ or acute ethanol exposure enhances ASIC1a protein degradation via the autophagy-lysosome pathway.

ASIC1 expression might play a role in vasoconstriction during pulmonary hypertension.

\section{Inflammatory Cytokines}

Our recent studies have concentrated on determining the relationship between inflammatory cytokines and ASIC1a expression in rheumatoid arthritis (RA). The results showed that several pro-inflammatory cytokines, including interleukin-6 (IL-6) (Zhou et al., 2015), IL-1 $\beta$
(Zhou et al., 2018), and tumor necrosis factor- $\alpha$ (TNF- $\alpha$ ) (Zhou et al., 2018) upregulate the levels of ASIC1a in a time- and dose-dependent manner in articular chondrocytes. Moreover, this effect was partially reversed by pretreating the cells with pyrrolidine dithiocarbamate (PDTC), an inhibitor of nuclear factor kappa $B(\mathrm{NF}-\kappa \mathrm{B})$, indicating that pro-inflammatory cytokines induced the upregulation of ASIC1a in articular chondrocytes mainly through the NF- $\mathrm{B}$ signaling pathway. 
NF- $\mathrm{B}$ is an evolutionarily conserved transcription factor involved in the expression of genes that play a critical role in various biological processes, including immune response, inflammation, proliferation, and apoptosis (Mitchell and Carmody, 2018). For instance, IL-1 $\beta$ and TNF- $\alpha$ play a role in ASIC1a protein synthesis through the NF- $\mathrm{KB}$ signaling pathway in the following ways (Zhou et al., 2018): (1) Co-expression of ASIC1a and NF-KB p65 was high in articular cartilage tissues, especially in AA rats, an experimental animal model of RA; (2) IL- $1 \beta$ or TNF- $\alpha$ increased the translocation of NF- $\mathrm{KB}$ p 65 to the nucleus in a time-dependent manner; (3) IL-1 $\beta$ - or TNF$\alpha$-induced ASIC1a expression was partially abrogated by PDTC; (4) IL-1 $\beta$ or TNF- $\alpha$ enhanced the activity of the ASICla gene promoter by increasing the DNA-binding activities of NF- $\mathrm{kB}$, which could be inhibited by PDTC. These results demonstrate that NF- $\mathrm{kB}$ activation is involved in the synthesis of the ASIC1a protein induced by IL- $1 \beta$ or TNF- $\alpha$.

\section{Neurotrophins}

Nerve growth factor (NGF), which regulates cell development and proliferation, has recently been identified as a mediator of the inflammatory response (Rocco et al., 2018). Similar to the regulatory effect of the pro-inflammatory cytokines on ASIC1a expression, NGF was also observed to increase mRNA and protein expression of ASIC1a in a dose- and time-dependent manner in chondrocytes (Wei et al., 2020). The NF-אB signaling pathway was also found to be involved in NGF governing the expression of ASIC1a (Wei et al., 2020).

Brain-derived neurotrophic factor (BDNF) and its neurotrophin receptor, TrkB, play important roles in neuronal plasticity and in the pathophysiology of various brain disorders. It has been recently proposed that BDNF/TrkB signaling is involved in regulation of the ASIC1a membrane trafficking process (Duan et al., 2012). Activation of phosphoinositide 3-kinase (PI3K) has been reported to promote the membrane trafficking of voltage-dependent $\mathrm{Ca}^{2+}$ channels (Viard et al., 2004). Moreover, activation of the PI3K/Akt signaling pathway promotes both the total expression and the number of ENaCs present in the membrane (Qi et al., 2014). The PI3K/Akt signaling pathway is also involved in the membrane trafficking process of ASIC1a, similar to its effect on ENaCs. Specifically, activation of TrkB by BDNF stimulated the intracellular PI3Kprotein kinase B/Akt pathway, induced ASIC1a phosphorylation, and targeted the neuronal surface in both rat spinal dorsal horn neurons and heterologous cell cultures. However, the co-administration of PI3K and Akt inhibitors attenuated the process, indicating a critical role of the PI3K/Akt pathway in BDNF-mediated upregulation of ASIC1a membrane trafficking. Further research confirmed the stimulatory effect of BDNF on the cell surface expression of ASIC1a, which was eliminated by mutation of the ASIC1a cytoplasmic residue Ser-25 (Duan et al., 2012), suggesting that Ser-25 is a functionally relevant phosphorylation site.

A similar signaling pathway is also involved in the process of platelet-derived growth factor (PDGF), regulating the expression and membrane trafficking of ASICla (Zuo et al., 2019). The expression and membrane trafficking of ASICla protein were remarkably increased in PDGF-stimulated hepatic stellate cells. However, these effects could be prevented by inhibiting activation of the PI3K/Akt signaling pathway with the inhibitor LY294002 (Zuo et al., 2019), revealing that PDGF stimulated the expression and membrane trafficking of ASICla via the PI3K/Akt pathway.

\section{Hormones}

Experimental and clinical data support a pathogenic role of estrogen metabolism and deficiency in RA (Islander et al., 2011; Dupuis et al., 2018). Our recent study demonstrated that ASICla is involved in the mechanism underlying estrogen replacement therapy in RA. It has been shown that the viability of chondrocytes was improved by pretreating the cells with PcTx1, an inhibitor of ASIC1a. Similar to the effect of PcTx1, pretreatment with $\beta$-estradiol also improved cell viability. The combined effect of $\beta$-estradiol and PcTx1 on the cells did not show an additive effect. Moreover, the effect was similar to that observed when PcTx1 was administered alone, suggesting that inhibiting ASIC1a is likely to involve $\beta$-estradiol-mediated protection. Furthermore, $\beta$-estradiol was able to downregulate the expression of ASIC1a protein through estrogen receptor $\alpha$ $(\mathrm{ER} \alpha)$ and protect the chondrocytes from acid-induced damage and apoptosis (Song et al., 2020). Further studies indicate that downregulation of ASICla protein expression can be attributed to $\beta$-estradiol, which promotes the degradation of ASICla protein through the autophagy-lysosomal pathway (Song et al., 2020). These findings suggest that $\beta$-estradiol has the potential to be developed as a novel strategy for the treatment of RA by downregulating ASIC1a protein expression. Activation of ASIC1a induced by tissue acidification, a salient feature of cerebral ischemia, plays a vital role in the progression of ischemia (Xiong et al., 2004; Li et al., 2016a). Recent in vitro and in vivo studies suggested that $\beta$-estradiol can protect neurons against the acidosis-mediated neurotoxicity and ischemic brain injury, possibly by suppressing ASICla protein expression (Zhou et al., 2019a). $\beta$-estradiol reduced the protein expression of ASIC1a in cerebral ischemia by promoting protein degradation through $\mathrm{ER} \alpha$, similar to its effect on ASIC1a protein expression in RA (Zhou et al., 2019a). These results highlight a novel mechanism underlying the protective effect of $\beta$-estradiol in tissue acidification-related diseases.

Insulin participates in the neuronal function by modulating expression of the various ion channels and neurotransmitter receptors on the cell surface (Wan et al., 1997; Skeberdis et al., 2001). Additionally, insulin was recently identified as a regulator of the ASIC1a membrane trafficking (Chai et al., 2010) and can maintain a low level of ASIC1a on the plasma membrane. In contrast, intracellular ASIC1a was transported to the cell surface during insulin deficiency, leading to an increase in ASIC1a expression on the membrane (Chai et al., 2010).

\section{Drugs}

Non-steroidal anti-inflammatory drugs (NSAIDs) are used to treat the inflammation- and pain-related disorders. Accumulating evidence links certain aspects of NSAID pharmacology with ASICs (Voilley et al., 2001; Sun et al., 2014). ASIC1 expression was upregulated in the nucleus 
pulposus cells due to chronic degeneration induced by the acidic conditions, and this effect was attenuated by treating the cells with ibuprofen, a widely used NSAID (Sun et al., 2014). Similarly, aspirin, another classic NSAID, was reported to inhibit the upregulation of ASIC1a mRNA and protein expression in the chondrocytes of AA rats (Wu et al., 2019).

Amiloride, a non-specific blocker of ASICs, is used to study the functions of ASICs (Leng and Xiong, 2013). Our previous study demonstrated that protein expression of ASIC1a was upregulated in the chondrocytes of AA rats, which was reversed by treating the cells with amiloride (Wu et al., 2019). Another study revealed that the increased expression of ASIC1 in the nucleus pulposus cells of the human intervertebral disc during degeneration was inhibited by amiloride treatment at a concentration of $1 \mathrm{mmol} / \mathrm{L}$ (Sun et al., 2014). Further research is needed to explore the effect of amiloride on the expression of ASIC1a at lower concentrations, since $1 \mathrm{mmol} / \mathrm{L}$ is an extremely high concentration for in vitro experiments.

PcTx1, the peptide toxin obtained from spiders, is a gating modifier of ASIC1a and has been widely used to explore the functions of ASIC1a (Leng and Xiong, 2013). Our previous studies have confirmed that PcTx1 reversed the enhancing effect of extracellular acidification on ASIC1a protein and mRNA expression in articular chondrocytes (Gao et al., 2019; Wu et al., 2019). Similarly, the increased expression and membrane trafficking of ASIC1a induced by PDGF stimulation were inhibited by PcTx1 (Zuo et al., 2019).

Omeprazole, a well-known proton-pump inhibitor, is frequently prescribed for the treatment of peptic ulcers through the anti-gastric mechanism mediated by acid secretion. Thongon et al. (2014) recently demonstrated that omeprazole enhanced ASIC1a expression in Caco-2 cells, leading to the inhibition of paracellular $\mathrm{Mg}^{2+}$ absorption via a $\mathrm{Ca}^{2+}$-dependent pathway.

Ginsenoside (GS)-Rd, the major active compound present in Panax ginseng, has neuroprotective effects against ischemic stroke (Nabavi et al., 2015; Zhang et al., 2016). A possible link between the neuroprotective effect of GS-Rd and ASICla activity has been hypothesized, since the $\mathrm{pH}$ of brain tissue can usually drop below 6.0 during severe ischemia (Wang et al., 2015). The rat middle cerebral artery occlusion (MCAO) model was used to investigate the effects of GS-Rd on the expression of ASICs in ischemic stroke. The results showed that the mRNA and protein expression levels of ASIC1a and ASIC2a were remarkably increased after stimulation with GS-Rd in the MCAO model. Pretreatment with GS-Rd not only attenuated ASIC1a upregulation, but also promoted ASIC2a expression (Zhang et al., 2012). These results indicate the neuroprotective effects of GS-Rd following cerebral ischemia may be related to its differential regulation in ASIC1a and ASIC2a expression.

\section{MicroRNAs}

Over the past decade, microRNA has emerged as an important group of regulatory molecules in controlling ion channels (Gross and Tiwari, 2018). A recent study by Zha et al. explored the effects of several miRNAs on the expression of ASIC1a, and they found that miR-144 and -149 reduced ASIC1a expression while let-7 increased ASIC1a protein expression (Jiang and Zha, 2017). In a subsequent study, it was confirmed that miR-149 targets the $3^{\prime}$-untranslated region of ASIC1a to regulate protein expression (Jiang and Zha, 2017). Collectively, further studies may provide an alternative to manipulate the expression of ASIC1a in acidosisrelated diseases.

\section{Effector Proteins}

Annexin II light chain p11, a member of the S100 family of small and dimeric EF-hand $\mathrm{Ca}^{2+}$-binding proteins, has been demonstrated to control the number of ENaCs present in the membrane via the exocytic pathway (Cheung et al., 2019). Considering that ASICs and ENaCs are both members of the DEG/ENaC gene family (Boscardin et al., 2016), it is rational to assume that p11 may have a regulatory effect on ASICs. Therefore, it was not surprising that p11 physically interacted with the N-terminus of ASIC1a (Donier et al., 2005). Moreover, an interaction between p11 and ASIC1a was demonstrated by immunoprecipitation in rat dorsal root ganglion in vivo (Donier et al., 2005). Furthermore, the co-expression of p11 and ASIC1a in CHO-K1 cells led to a twofold increase in ASIC1a expression on the plasma membrane (Donier et al., 2005). These results indicated that 11 might have a role in regulating ASIC1a expression on the plasma membrane. Additionally, further research is required to confirm whether the underlying mechanism by which p11 regulates ASIC1a expression on the plasma membrane is similar to that of regulating $\mathrm{ENaC}$ expression through the exocytic pathway.

Protein interacting with $\mathrm{C}$ kinase 1 (PICK1) is a peripheral membrane protein that regulates trafficking of diverse membrane proteins (Li et al., 2016b). Several studies have indicated a close connection between PICK1 and ASIC1a. First, ASIC1a has been shown to interact with the PDZ domain of PICK1 through its C-terminus, and this interaction changes the subcellular distribution of ASIC1a (Duggan et al., 2002; Hruska-Hageman et al., 2002). Second, PICK1 overexpression increases the expression of ASIC1a on the cell surface, which depends on the BAR domain of PICK1 (Jin et al., 2010). Third, knockout of the gene PICK1 is attributed to the decreased expression of ASIC1a and ASIC2a proteins on the plasma membrane (Hu et al., 2010). Fourth, the link between PICK1 and ASIC1a is regulated by protein kinases, including protein kinase $(\mathrm{PK}) \mathrm{A}$ and $\mathrm{PKC}$ (Leonard et al., 2003; Hu et al., 2010). These findings provide compelling evidence that blocking the link between ASIC1a and PICK1 can be used as a treatment for ASIC1a-mediated diseases.

RhoA, a small $G$ protein of the Rho family, has been demonstrated to promote $\mathrm{ENaC}$ trafficking to the plasma membrane, thereby increasing its activity (Staruschenko et al., 2004; Pochynyuk et al., 2007). Similarly, activation of RhoA increased ASIC1a expression on the plasma membrane and enhanced store-operated $\mathrm{Ca}^{2+}$ entry in the pulmonary arterial smooth muscle cells (Herbert et al., 2018), which might help improve our understanding of the vital role of RhoA in the pathogenesis of pulmonary hypertension.

Serum- and glucocorticoid-inducedkinase-1 (SGK1) plays an important role in the modulation of ion channels and regulation of ENaCs (Lang and Pearce, 2016). Recently, SGK1.1, a spliced isoform of SGK1, has been found to downregulate the activity of 
neuronal ASIC1, at least in part, by decreasing the expression of the channels on the plasma membrane (Arteaga et al., 2008).

There is extensive amount of evidence that suggests both $\mathrm{ENaCs}$ and ASICs are regulated by clathrin-dependent endocytosis (Wang et al., 2006; Zeng et al., 2014). Clathrin adapter protein 2 (AP2), a heterotetrameric complex containing $\alpha \beta_{2} \mu_{2} \sigma_{2}$ subunits, links membrane proteins to clathrin, which initiates clathrin assembly at the cell surface (McMahon and Boucrot, 2011). Tyrphostin A23, a pharmacological clathrinmediated endocytosis inhibitor, reduced the association of clathrin with AP2 in the membrane (Wang et al., 2016), and increased the expression level of ASIC1a on the membrane in both mouse cortical neurons and heterologous cells, indicating a regulative role of clathrin-mediated endocytosis in the surface density of ASIC1a (Zeng et al., 2013). Moreover, knockdown of AP2 $\mu 2$ (a core subunit of the AP2 complex) also enhanced the surface density of ASIC1a, indicating a critical role of the AP2 complex in ASICla internalization.

Dynamin is a large GTP are responsible for diverse cellular processes, including endocytosis, and plays a crucial role in vesicle scission after cargo internalization (Antonny et al., 2016). Blocking the constitutive endocytosis of ASICla with the dominant-negative dynamin1 $\mathrm{K} 44 \mathrm{~A}$ or dynasore, a smallmolecule dynamin inhibitor, increased the surface density of ASIC1a protein (Zeng et al., 2013). These results indicate that ASIC1a undergoes constitutive clathrin- and dynamindependent endocytosis, resulting in downregulation of ASIC1a expression at the cell surface.

\section{Chemicals}

It is now widely known that acute ethanol administration has neuroprotective effects during cerebral ischemia (Wang et al., 2012). Tissue acidification and its associated activation of ASIC1a are common features of cerebral ischemia (Xiong et al., 2004). Our recent study showed that the neuroprotective effect of ethanol might be related to the regulation of ASICla expression in neurons against acidosis-induced neurotoxicity (Zhou et al., 2019b). It has been indicated that acute treatment of neurons with ethanol decreased ASICla protein expression and acid-induced $\left[\mathrm{Ca}^{2+}\right]$ elevation (Zhou et al., 2019b). Further evidence suggests that the downregulation of ASICla protein was mediated by degradation of the protein via the autophagy-lysosome pathway (Zhou et al., 2019b).

Hydrogen peroxide $\left(\mathrm{H}_{2} \mathrm{O}_{2}\right)$ is an endogenous reactive oxygen species that contributes to oxidative stress (Murphy and Friedman, 2019). A previous study showed that the application of oxidants inhibited ASIC1a currents in cultured mouse cortical neurons (Chu et al., 2006). More importantly, $\mathrm{H}_{2} \mathrm{O}_{2}$ has been reported to affect the links between the three subunits of ASIC1a (Zha et al., 2009). Previous studies have demonstrated that inter-subunit disulfide bonds could form intracellularly between ion channel subunits, including ASIC1a. Inter-subunit disulfide bonds can produce ASICla complexes that are larger than the trimers (Zha et al., 2009). Given that ASICla presents on the cell membrane as a trimer, these cytoplasmic ASICla complexes that are larger than the trimers combine by disulfide bonds to affect the transport of ASICla to the cell membrane, leading to a decrease in their cell surface expression. By targeting the C-terminal cysteines, $\mathrm{H}_{2} \mathrm{O}_{2}$ increases the inter-subunit disulfide bond formation, leading to the reduced expression of ASIC1a located on the cell membrane and reduced $\mathrm{H}^{+}$-gated current (Zha et al., 2009).

Nitric oxide $(\mathrm{NO})$ is a free radical signaling molecule that regulates numerous physiological and pathological conditions. NO signaling has been shown to increase the expression of transient receptor potential vanilloid type 2, a calcium channel, and its trafficking to the plasma membrane via a PI3K dependent pathway (Maksoud et al., 2019), suggesting that NO signaling has the potential to regulate the expression of membrane proteins. Recently, differential regulatory effects of $\mathrm{NO}$ signaling on ASICla expression in the prefrontal cortex (PFC) and hippocampus have been confirmed. Microinjection of S-nitroso$\mathrm{N}$-acetyl-D, L-penicillamine, an NO donor, upregulated the expression of ASICla in the PFC and downregulated its expression in the hippocampus. In contrast, 7-nitroindazole, an nNOS inhibitor, showed the opposite effect on the regulation of ASIC1a expression in the PFC and hippocampus (Li et al., 2019).

\section{N-Glycosylation}

$N$-glycosylation, a ubiquitous protein modification, alters the molecular and functional features of glycoproteins and is involved in various physiological processes and diseases. A large number of studies have demonstrated that $\mathrm{N}$-glycosylation of the extracellular domains of some membrane proteins is important for maturation and apical location of these proteins (Vagin et al., 2009; Moremen et al., 2012). A close relationship between $\mathrm{N}$-glycosylation and ASICla membrane expression has been observed in several studies: (1) there was a high proportion of glycosylated ASIC1a on the surface of $\mathrm{CHO}$ cells and hippocampal neurons, indicating that mature ASIC1a was preferentially transported to the cell surface (Jing et al., 2012); (2) inhibition of glycosylation with tunicamycin reduced ASIC1a surface transport (Jing et al., 2012); (3) disrupting the interaction between the first transmembrane domain and the thumb of ASIC1a altered ASIC1a folding, inhibited its glycosylation, and reduced its surface trafficking (Jing et al., 2011).

\section{CONCLUSION}

Recent studies have demonstrated that ASIC1a plays a crucial role in the occurrence and development of diseases related to the central and peripheral nervous system, and it is considered to be a potential therapeutic target. Elucidating the factors and the underlying molecular mechanisms affecting ASIC1a protein expression and membrane trafficking is necessary to better understand the role of ASIC1a in various pathophysiological conditions. This review focused on these topics and summarized the currently known factors that affect ASIC1a expression and membrane transfer and the possible underlying mechanism of ASIC1a synthesis and degradation, as well as membrane trafficking. Several studies suggest that ASIC1a is a promising therapeutic target in acidosis-related diseases. 
In order to explore whether ASIC1a can be a potential therapeutic target, several questions remain to be answered. These questions are related to (1) the dynamic changes in ASIC1a expression and membrane transfer during disease progression; (2) the contribution of ASIC1a synthesis, membrane transfer, and degradation in the pathogenesis of diseases; (3) the identification of novel drugs that specifically block ASIC1a and have fewer side effects than the conventional drugs (for example, amiloride can be structurally modified to improve the specific blocking of ASIC1a); and (4) isoform-specific membrane trafficking motifs and the related accessory proteins of ASIC1a. All of these require further confirmation.

In conclusion, although multiple lines of evidence suggest that ASIC1a is an important contributor to multiple acidosis-related diseases and various factors affect its expression and membrane

\section{REFERENCES}

Antonny, B., Burd, C., De Camilli, P., Chen, E., Daumke, O., Faelber, K., et al. (2016). Membrane fission by dynamin: what we know and what we need to know. EMBO J. 35, 2270-2284. doi: 10.15252/embj.201694613

Arteaga, M., Coric, T., Straub, C., and Canessa, C. (2008). A brain-specific SGK1 splice isoform regulates expression of ASIC1 in neurons. Proc. Natl. Acad. Sci. U.S.A. 105, 4459-4464. doi: 10.1073/pnas.0800958105

Boscardin, E., Alijevic, O., Hummler, E., Frateschi, S., and Kellenberger, S. (2016). The function and regulation of acid-sensing ion channels (ASICs) and the epithelial $\mathrm{Na}(+)$ channel $(\mathrm{ENaC})$ : IUPHAR Review 19. Br. J. Pharmacol. 173, 2671-2701. doi: 10.1111/bph.13533

Chai, S., Li, M., Branigan, D., Xiong, Z. G., and Simon, R. P. (2010). Activation of acid-sensing ion channel 1a (ASIC1a) by surface trafficking. J. Biol. Chem. 285, 13002-13011. doi: 10.1074/jbc.M109.086041

Cheng, Y. R., Jiang, B. Y., and Chen, C. C. (2018). Acid-sensing ion channels: dual function proteins for chemo-sensing and mechano-sensing. J. Biomed. Sci. 25:46. doi: 10.1186/s12929-018-0448-y

Cheung, T., Ismail, N., Moir, R., Arora, N., McDonald, F., and Condliffe, S. (2019). Annexin II Light Chain p11 Interacts With ENaC to Increase Functional Activity at the Membrane. Front. Physiol. 10:7. doi: 10.3389/fphys.2019.00007

Chu, X. P., Close, N., Saugstad, J., and Xiong, Z. G. (2006). ASICla-specific modulation of acid-sensing ion channels in mouse cortical neurons by redox reagents. J. Neurosci. 26, 5329-5339. doi: 10.1523/jneurosci.0938-06.2006

Dai, B., Zhu, F., Chen, Y., Zhou, R., Wang, Z., Xie, Y., et al. (2017). ASIC1a Promotes Acid-Induced Autophagy in Rat Articular Chondrocytes through the AMPK/FoxO3a Pathway. Int. J. Mol. Sci. 18:2125. doi: 10.3390/ijms18102125

Damgaci, S., Ibrahim-Hashim, A., Enriquez-Navas, P., Pilon-Thomas, S., Guvenis, A., and Gillies, R. (2018). Hypoxia and acidosis: immune suppressors and therapeutic targets. Immunology 154, 354-362. doi: 10.1111/imm.12917

Deval, E., Gasull, X., Noël, J., Salinas, M., Baron, A., Diochot, S., et al. (2010). Acid-sensing ion channels (ASICs): pharmacology and implication in pain. Pharmacol. Ther. 128, 549-558.

Donier, E., Rugiero, F., Okuse, K., and Wood, J. N. (2005). Annexin II light chain p11 promotes functional expression of acid-sensing ion channel ASIC1a. J. Biol. Chem. 280, 38666-38672. doi: 10.1074/jbc.M505981200

Duan, B., Liu, D. S., Huang, Y., Zeng, W. Z., Wang, X., Yu, H., et al. (2012). PI3kinase/Akt pathway-regulated membrane insertion of acid-sensing ion channel 1a underlies BDNF-induced pain hypersensitivity. J. Neurosci. 32, 6351-6363. doi: 10.1523/JNEUROSCI.4479-11.2012

Duan, B., Wu, L. J., Yu, Y. Q., Ding, Y., Jing, L., Xu, L., et al. (2007). Upregulation of acid-sensing ion channel ASICla in spinal dorsal horn neurons contributes to inflammatory pain hypersensitivity. J. Neurosci. 27, 11139-11148. doi: 10.1523/ jneurosci.3364-07.2007

Duggan, A., Garcia-Anoveros, J., and Corey, D. (2002). The PDZ domain protein PICK1 and the sodium channel $\mathrm{BNaCl}$ interact and localize at mechanosensory terminals of dorsal root ganglion neurons and dendrites of central neurons. J. Biol. Chem. 277, 5203-5208. doi: 10.1074/jbc.M104748200 transfer, additional clinical studies are needed to confirm its therapeutic efficacy and safety.

\section{AUTHOR CONTRIBUTIONS}

YX and FC designed this work and revised the manuscript. YX wrote the manuscript. Both authors contributed to the article and approved the submitted version.

\section{FUNDING}

This work was supported by the National Natural Science Foundation of China (Grant Number 81873986).

Dupuis, M., Conti, F., Maselli, A., Pagano, M., Ruggieri, A., Anticoli, S., et al. (2018). The Natural Agonist of Estrogen Receptor $\beta$ Silibinin Plays an Immunosuppressive Role Representing a Potential Therapeutic Tool in Rheumatoid Arthritis. Front. Immunol. 9:1903. doi: 10.3389/fimmu.2018.0 1903

Gao, W. F., Xu, Y. Y., Ge, J. F., and Chen, F. H. (2019). Inhibition of acid-sensing ion channel la attenuates acid-induced activation of autophagy via a calcium signaling pathway in articular chondrocytes. Int. J. Mol. Med. 43, 1778-1788. doi: $10.3892 /$ ijmm.2019.4085

Gross, C., and Tiwari, D. (2018). Regulation of Ion Channels by MicroRNAs and the Implication for Epilepsy. Curr. Neurol. Neurosci. Rep. 18:60. doi: 10.1007/ s11910-018-0870-2

Herbert, L. M., Resta, T. C., and Jernigan, N. L. (2018). RhoA increases ASIC1a plasma membrane localization and calcium influx in pulmonary arterial smooth muscle cells following chronic hypoxia. Am. J. Physiol. Cell Physiol. 314, C166C176. doi: 10.1152/ajpcell.00159.2017

Hruska-Hageman, A., Wemmie, J., Price, M., and Welsh, M. (2002). Interaction of the synaptic protein PICK1 (protein interacting with $\mathrm{C}$ kinase 1) with the non-voltage gated sodium channels BNC1 (brain Na+ channel 1) and ASIC (acid-sensing ion channel). Biochem. J. 361, 443-450. doi: 10.1042/0264-6021: 3610443

Hu, Z. L., Huang, C., Fu, H., Jin, Y., Wu, W. N., Xiong, Q. J., et al. (2010). Disruption of PICK1 attenuates the function of ASICs and PKC regulation of ASICs. Am. J. Physiol. Cell Physiol. 299, C1355-C1362. doi: 10.1152/ajpcell. 00569.2009

Islander, U., Jochems, C., Lagerquist, M., Forsblad-d'Elia, H., and Carlsten, H. (2011). Estrogens in rheumatoid arthritis; the immune system and bone. Mol. Cell. Endocrinol. 335, 14-29. doi: 10.1016/j.mce.2010.05.018

Jernigan, N., Herbert, L., Walker, B., and Resta, T. (2012). Chronic hypoxia upregulates pulmonary arterial ASIC1: a novel mechanism of enhanced storeoperated $\mathrm{Ca} 2+$ entry and receptor-dependent vasoconstriction. Am. J. Physiol. Cell Physiol. 302, C931-C940. doi: 10.1152/ajpcell.00332.2011

Jiang, Y. Q., and Zha, X. M. (2017). miR-149 reduces while let-7 elevates ASIC1a expression in vitro. Int. J.Physiol. Pathophysiol. Pharmacol. 9, 147-152.

Jin, W., Shen, C., Jing, L., Zha, X. M., and Xia, J. (2010). PICK1 regulates the trafficking of ASICla and acidotoxicity in a BAR domain lipid bindingdependent manner. Mol. Brain 3:39. doi: 10.1186/1756-6606-3-39

Jing, L., Chu, X. P., Jiang, Y. Q., Collier, D., Wang, B., Jiang, Q., et al. (2012). $\mathrm{N}$-glycosylation of acid-sensing ion channel 1a regulates its trafficking and acidosis-induced spine remodeling. J. Neurosci. 32, 4080-4091. doi: 10.1523/ jneurosci.5021-11.2012

Jing, L., Jiang, Y. Q., Jiang, Q., Wang, B., Chu, X. P., and Zha, X. M. (2011). The interaction between the first transmembrane domain and the thumb of ASICla is critical for its N-glycosylation and trafficking. PLoS One 6:e26909. doi: 10.1371/journal.pone.0026909

Lang, F., and Pearce, D. (2016). Regulation of the epithelial Na+ channel by the mTORC2/SGK1 pathway. Nephrol. Dial. Transplant. 31, 200-205. doi: 10.1093/ ndt/gfv270 
Leng, T. D., and Xiong, Z. G. (2013). The pharmacology and therapeutic potential of small molecule inhibitors of acid-sensing ion channels in stroke intervention. Acta Pharmacol. Sin. 34, 33-38. doi: 10.1038/aps.2012.81

Leonard, A., Yermolaieva, O., Hruska-Hageman, A., Askwith, C., Price, M., Wemmie, J., et al. (2003). cAMP-dependent protein kinase phosphorylation of the acid-sensing ion channel-1 regulates its binding to the protein interacting with C-kinase-1. Proc. Natl. Acad. Sci. U.S.A. 100, 2029-2034. doi: 10.1073/pnas. 252782799

Li, M. H., Leng, T. D., Feng, X. C., Yang, T., Simon, R., and Xiong, Z. G. (2016a). Modulation of Acid-sensing Ion Channel 1a by Intracellular $\mathrm{pH}$ and Its Role in Ischemic Stroke. J. Biol. Chem. 291, 18370-18383. doi: 10.1074/jbc.M115. 713636

Li, Y. H., Zhang, N., Wang, Y. N., Shen, Y., and Wang, Y. (2016b). Multiple faces of protein interacting with C kinase 1 (PICK1): Structure, function, and diseases. Neurochem. Int. 98, 115-121. doi: 10.1016/j.neuint.2016.0 3.001

Li, M. M., Zhou, P., Chen, X. D., Xu, H. S., Wang, J., Chen, L., et al. (2019). $\mathrm{NO}$ in the dPAG modulates panic-like responses and ASICla expression in the prefrontal cortex and hippocampus in mice. Biochem. Biophys. Res. Commun. 511, 274-279. doi: 10.1016/j.bbrc.2019.02.020

Maksoud, M., Tellios, V., An, D., Xiang, Y. Y., and Lu, W. Y. (2019). Nitric oxide upregulates microglia phagocytosis and increases transient receptor potential vanilloid type 2 channel expression on the plasma membrane. Glia 67, 22942311. doi: $10.1002 /$ glia. 23685

Matricon, J., Muller, E., Accarie, A., Meleine, M., Etienne, M., Voilley, N., et al. (2013). Peripheral contribution of NGF and ASIC1a to colonic hypersensitivity in a rat model of irritable bowel syndrome. Neurogastroenterol. Motil. 25, 740-754e. doi: 10.1111/nmo.12199

McMahon, H., and Boucrot, E. (2011). Molecular mechanism and physiological functions of clathrin-mediated endocytosis. Nat. Rev. Mol. Cell Biol. 12, $517-$ 533. doi: $10.1038 / \mathrm{nrm} 3151$

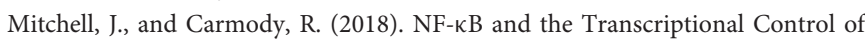
Inflammation. Int. Rev. Cell. Mol. Biol. 335, 41-84. doi: 10.1016/bs.ircmb.2017. 07.007

Moremen, K., Tiemeyer, M., and Nairn, A. (2012). Vertebrate protein glycosylation: diversity, synthesis and function. Nat. Rev. Mol. Cell Biol. 13, 448-462. doi: $10.1038 /$ nrm 3383

Murphy, E., and Friedman, A. (2019). Hydrogen peroxide and cutaneous biology: Translational applications, benefits, and risks. J. Am. Acad. Dermatol. 81, 1379-1386. doi: 10.1016/j.jaad.2019.05.030

Nabavi, S., Sureda, A., Habtemariam, S., and Nabavi, S. (2015). Ginsenoside Rd and ischemic stroke; a short review of literatures. J. Ginseng. Res. 39, 299-303. doi: 10.1016/j.jgr.2015.02.002

Pochynyuk, O., Staruschenko, A., Bugaj, V., Lagrange, L., and Stockand, J. (2007). Quantifying RhoA facilitated trafficking of the epithelial $\mathrm{Na}+$ channel toward the plasma membrane with total internal reflection fluorescence-fluorescence recovery after photobleaching. J. Biol. Chem. 282, 14576-14585. doi: 10.1074/ jbc.M701348200

Qian, X. W., Zhang, Y. H., Tao, J. J., Niu, R. W., Song, S. J., Wang, C., et al. (2020). Acidosis induces synovial fibroblasts to release vascular endothelial growth factor via acid-sensitive ion channel 1a. Lab. Invest. 21. doi: 10.1038/s41374020-0423-6

Qi, D., He, J., Wang, D., Deng, W., Zhao, Y., Ye, Y., et al. (2014). 17ßestradiol suppresses lipopolysaccharide-induced acute lung injury through PI3K/Akt/SGK1 mediated up-regulation of epithelial sodium channel $(\mathrm{ENaC})$ in vivo and in vitro. Respir. Res. 15:159. doi: 10.1186/s12931-01 4-0159-1

Radu, B., Banciu, A., Banciu, D., and Radu, M. (2016). Acid-Sensing Ion Channels as Potential Pharmacological Targets in Peripheral and Central Nervous System Diseases. Adv. Protein Chem. Struct. Biol. 103, 137-167. doi: 10.1016/bs.apcsb. 2015.10.002

Rocco, M., Soligo, M., Manni, L., and Aloe, L. (2018). Nerve Growth Factor: Early Studies and Recent Clinical Trials. Curr. Neuropharmacol. 16, 1455-1465. doi: 10.2174/1570159x16666180412092859

Skeberdis, V., Lan, J., Zheng, X., Zukin, R., and Bennett, M. (2001). Insulin promotes rapid delivery of N-methyl-D- aspartate receptors to the cell surface by exocytosis. Proc. Natl. Acad. Sci. U.S.A 98, 3561-3566. doi: 10.1073/pnas. 051634698
Song, S. J., Tao, J. J., Li, S. F., Qian, X. W., Niu, R. W., Wang, C., et al. (2020). $17 \beta$-estradiol attenuates rat articular chondrocyte injury by targeting ASIClamediated apoptosis. Mol. Cell. Endocrinol. 505:110742. doi: 10.1016/j.mce.2020. 110742

Staruschenko, A., Nichols, A., Medina, J., Camacho, P., Zheleznova, N., and Stockand, J. (2004). Rho small GTPases activate the epithelial $\mathrm{Na}(+)$ channel. J. Biol. Chem. 279, 49989-49994. doi: 10.1074/jbc.M409812200

Sun, X., Jin, J., Zhang, J. G., Qi, L., Braun, F., Zhang, X. D., et al. (2014). Expression of acid-sensing ion channels in nucleus pulposus cells of the human intervertebral disk is regulated by non-steroid anti-inflammatory drugs. Acta Biochim. Biophys. Sin. 46, 774-781. doi: 10.1093/abbs/gmu067

Tan, J., Ye, X., Xu, Y., Wang, H., Sheng, M., and Wang, F. (2011). Acid-sensing ion channel 1a is involved in retinal ganglion cell death induced by hypoxia. Mol. Vis. 17, 3300-3308.

Thongon, N., Ketkeaw, P., and Nuekchob, C. (2014). The roles of acid-sensing ion channel 1a and ovarian cancer G protein-coupled receptor 1 on passive $\mathrm{Mg} 2+$ transport across intestinal epithelium-like Caco-2 monolayers. J. Physiol. Sci. 64, 129-139. doi: 10.1007/s12576-013-0301-8

Vagin, O., Kraut, J., and Sachs, G. (2009). Role of N-glycosylation in trafficking of apical membrane proteins in epithelia. Am. J. Physiol. Renal Physiol. 296, F459-F469. doi: 10.1152/ajprenal.90340.2008

Viard, P., Butcher, A., Halet, G., Davies, A., Nürnberg, B., Heblich, F., et al. (2004). PI3K promotes voltage-dependent calcium channel trafficking to the plasma membrane. Nat. Neurosci. 7, 939-946. doi: 10.1038/nn1300

Voilley, N., de Weille, J., Mamet, J., and Lazdunski, M. (2001). Nonsteroid antiinflammatory drugs inhibit both the activity and the inflammation-induced expression of acid-sensing ion channels in nociceptors. J. Neurosci. 21, 80268033. doi: 10.1523/jneurosci.21-20-08026.2001

Waldmann, R., and Lazdunski, M. (1998). H(+)-gated cation channels: neuronal acid sensors in the NaC/DEG family of ion channels. Curr. Opin. Neurobiol. 8 , 418-424. doi: 10.1016/s0959-4388(98)80070-6

Wan, Q., Xiong, Z. G., Man, H. Y., Ackerley, C. A., Braunton, J., Lu, W. Y., et al. (1997). Recruitment of functional GABA(A) receptors to postsynaptic domains by insulin. Nature 388, 686-690. doi: 10.1038/41792

Wang, C., Hu, T., Yan, X., Meng, T., Wang, Y., Wang, Q., et al. (2016). Differential Regulation of Clathrin and Its Adaptor Proteins during Membrane Recruitment for Endocytosis. Plant Physiol. 171, 215-229. doi: 10.1104/pp.15.01716

Wang, F., Wang, Y., Geng, X., Asmaro, K., Peng, C., Sullivan, J., et al. (2012). Neuroprotective effect of acute ethanol administration in a rat with transient cerebral ischemia. Stroke 43, 205-210. doi: 10.1161/strokeaha.111.629576

Wang, H., Traub, L., Weixel, K., Hawryluk, M., Shah, N., Edinger, R., et al. (2006). Clathrin-mediated endocytosis of the epithelial sodium channel: role of epsin. J. Biol. Chem. 281, 14129-14135. doi: 10.1074/jbc.M512511200

Wang, Y. Z., Wang, J. J., Huang, Y., Liu, F., Zeng, W. Z., Li, Y., et al. (2015). Tissue acidosis induces neuronal necroptosis via ASICla channel independent of its ionic conduction. Elife 4:e05682. doi: 10.7554/eLife.05682

Wei, X., Sun, C., Zhou, R. P., Ma, G. G., Yang, Y., Lu, C., et al. (2020). Nerve growth factor promotes ASIC1a expression via the NF- $\kappa B$ pathway and enhances acidinduced chondrocyte apoptosis. Int. Immunopharmacol. 82:106340. doi: 10. 1016/j.intimp.2020.106340

Wu, J., Xu, Y., Jiang, Y. Q., Xu, J., Hu, Y., and Zha, X. M. (2016). ASIC subunit ratio and differential surface trafficking in the brain. Mol. Brain 9:4. doi: 10. 1186/s13041-016-0185-7

Wu, X., Ren, G., Zhou, R., Ge, J., and Chen, F. H. (2019). The role of $\mathrm{Ca}^{2+}$ in acid-sensing ion channel la-mediated chondrocyte pyroptosis in rat adjuvant arthritis. Lab. Invest. 99, 499-513. doi: 10.1038/s41374-018-0135-3

Xiong, Z. G., Zhu, X. M., Chu, X. P., Minami, M., Hey, J., Wei, W. L., et al. (2004). Neuroprotection in ischemia: blocking calcium-permeable acid-sensing ion channels. Cell 118, 687-698. doi: 10.1016/j.cell.2004.08.026

Yang, Y., Yu, Y., Cheng, J., Liu, Y., Liu, D., Wang, J., et al. (2012). Highly conserved salt bridge stabilizes rigid signal patch at extracellular loop critical for surface expression of acid-sensing ion channels. J. Biol. Chem. 287, 14443-14455. doi: 10.1074/jbc.M111.334250

Yingjun, G., and Xun, Q. (2013). Acid-sensing ion channels under hypoxia. Channels 7, 231-237. doi: 10.4161/chan.25223

Zeng, W. Z., Liu, D. S., and Xu, T. L. (2014). Acid-sensing ion channels: trafficking and pathophysiology. Channels 8, 481-487. doi: 10.4161/19336950. 2014.958382 
Zeng, W. Z., Liu, D. S., Duan, B., Song, X. L., Wang, X., Wei, D., et al. (2013). Molecular mechanism of constitutive endocytosis of Acid-sensing ion channel 1a and its protective function in acidosis-induced neuronal death. J. Neurosci. 33, 7066-7078. doi: 10.1523/jneurosci.5206-12.2013

Zha, X. M., Wang, R., Collier, D., Snyder, P., Wemmie, J., and Welsh, M. (2009). Oxidant regulated inter-subunit disulfide bond formation between ASICla subunits. Proc. Natl. Acad. Sci. U.S.A. 106, 3573-3578. doi: 10.1073/pnas. 0813402106

Zhang, G., Xia, F., Zhang, Y., Zhang, X., Cao, Y., Wang, L., et al. (2016). Ginsenoside Rd Is Efficacious Against Acute Ischemic Stroke by Suppressing Microglial Proteasome-Mediated Inflammation. Mol. Neurobiol. 53, 25292540. doi: 10.1007/s12035-015-9261-8

Zhang, Y., Zhou, L., Zhang, X., Bai, J., Shi, M., and Zhao, G. (2012). Ginsenoside-Rd attenuates TRPM7 and ASIC1a but promotes ASIC2a expression in rats after focal cerebral ischemia. Neurol. Sci. 33, 1125-1131. doi: 10.1007/s10072-0110916-6

Zhou, R. P., Dai, B. B., Xie, Y. Y., Wu, X. S., Wang, Z. S., Li, Y., et al. (2018). Interleukin-1 $\beta$ and tumor necrosis factor- $\alpha$ augment acidosis-induced rat articular chondrocyte apoptosis via nuclear factor-kappaB-dependent upregulation of ASIC1a channel. Biochim. Biophys. Acta Mol. Basis Dis. 1864, 162-177. doi: 10.1016/j.bbadis.2017.10.004

Zhou, R. P., Leng, T. D., Yang, T., Chen, F. H., Hu, W., and Xiong, Z. G. (2019a). $\beta$ Estradiol Protects Against Acidosis-Mediated and Ischemic Neuronal Injury by Promoting ASIC1a (Acid-Sensing Ion Channel 1a) Protein Degradation. Stroke 50, 2902-2911. doi: 10.1161/strokeaha.119.025940

Zhou, R. P., Leng, T. D., Yang, T., Chen, F. H., and Xiong, Z. G. (2019b). Acute Ethanol Exposure Promotes Autophagy-Lysosome
Pathway-Dependent ASICla Protein Degradation and Protects Against Acidosis-Induced Neurotoxicity. Mol. Neurobiol. 56, 3326-3340.

Zhou, R. P., Wu, X. S., Wang, Z. S., Ge, J. F., and Chen, F. H. (2015). Interleukin-6 enhances acid-induced apoptosis via upregulating acid-sensing ion channel 1a expression and function in rat articular chondrocytes. Int. Immunopharmacol. 29, 748-760. doi: 10.1016/j.intimp.2015.08.044

Zu, S. Q., Feng, Y. B., Zhu, C. J., Wu, X. S., Zhou, R. P., Li, G., et al. (2020). Acid-sensing ion channel 1a mediates acid-induced pyroptosis through calpain-2/calcineurin pathway in rat articular chondrocytes. Cell Biol. Int. 2020:32678496.doi: 10.1002/cbin.11422

Zuo, L., Zhu, Y., Hu, L., Liu, Y., Wang, Y., Hu, Y., et al. (2019). PI3-kinase/Akt pathway-regulated membrane transportation of acid-sensing ion channel 1a/Calcium ion influx/endoplasmic reticulum stress activation on PDGFinduced HSC Activation. J. Cell. Mol. Med. 23, 3940-3950. doi: 10.1111/jcmm. 14275

Conflict of Interest: The authors declare that the research was conducted in the absence of any commercial or financial relationships that could be construed as a potential conflict of interest.

Copyright (c) $2020 \mathrm{Xu}$ and Chen. This is an open-access article distributed under the terms of the Creative Commons Attribution License (CC BY). The use, distribution or reproduction in other forums is permitted, provided the original author(s) and the copyright owner(s) are credited and that the original publication in this journal is cited, in accordance with accepted academic practice. No use, distribution or reproduction is permitted which does not comply with these terms. 